\title{
Dynamic disruption simulation in large-scale urban rail transit systems ${ }^{\star}$
}

\author{
Steffen O.P. Blume ${ }^{1,2[0000-0002-7210-6352]}$, Michel-Alexandre \\ Cardin ${ }^{3[0000-0002-2337-1133]}$, and Giovanni Sansavini ${ }^{10000-0002-8801-9667]}$ \\ 1 Department of Mechanical and Process Engineering, ETH Zrich, Zrich, Switzerland \\ sansavig@ethz.ch \\ 2 Future Resilient Systems, Singapore-ETH Centre, Singapore \\ steffen.blume@frs.ethz.ch \\ 3 Dyson School of Design Engineering, Imperial College London, London, United \\ Kingdom \\ m. cardin@imperial.ac.uk
}

\begin{abstract}
We present a simulation-based approach to capture the interactions between train operations and passenger behavior during disruptions in urban rail transit systems. The simulation models the full disruption and recovery cycle. It is based on a discrete-event simulation framework to model the network vehicles movement. It is paired with an agent-based model to replicate passenger route choices and decisions during both the undisrupted and disrupted state of the system. We demonstrate that optimizing and flexibly changing the train dispatch schedules on specific routes reduces the impact of disruptions. Moreover, we show that demand uncertainty considerably changes the measures of performance during the disruption. However, the optimized schedule still outperforms the non-optimized schedule even under demand uncertainty. This work ties into our ongoing project to find flexible strategies to enhance the system resilience by explicitly incorporating uncertainties into the design of rail system architectures and operational strategies.
\end{abstract}

Keywords: Urban Rail · Disruption Simulation · Dynamic Transit Assignment.

\section{Introduction}

Many cities rely on large-scale metropolitan rail networks to transport millions of passengers each day. However, expanding complexity is taking its toll on their resilience to disruptions. Despite many operational and technological improvements, disruptions remain inevitable and are potentially more catastrophic to the ever-growing urban population. Moreover, the uncertainties associated with the system operations and passengers behavior during both undisrupted and

\footnotetext{
* This research was conducted under the Future Resilient Systems program at the Singapore-ETH Centre and funded by the National Research Foundation of Singapore.
} 
especially disrupted conditions make it ever more complex to understand the impact of disruptions. In designing pre-emptive and immediate disruption recovery control strategies, it is therefore imperative to consider the effects of control actions on passenger flows and assess how demand uncertainty propagates into the performance of the system during disruptions.

A large body of literature has been devoted to modeling passenger flows, train operations, their interactions, as well as schedule optimizations. To name a few, Cats [1] developed an agent-based dynamic transit assignment model and simulation platform known as BusMezzo that explicitly models public vehicles, passengers behavior, and specifically their interactions. Much work has followed, that builds on this simulation platform to assess the effects of real-time transit information $[2,3]$, or the planning of excess system capacity to reduce the impact of disruptions and congestion in [4] and [5], respectively. Moreover, other works have demonstrated the use of agent-based models for dynamic passenger assignment modelling of congestion dynamics [6] and station closures [7]. At the same time, other research has looked at schedule optimization during disruptions, focusing on system-wide rolling stock rescheduling [8] and the effects of passenger advice [9], train trip short-turning based on estimating the duration of a disruption [10], or train trip re-timing and changing stop sequences [11]. For further work on schedule optimization, we refer to the review on rail schedule optimization in [12].

Despite the exhaustive list of work, the effects of passenger demand uncertainty, together with controller action uncertainty, and imperfect vehicle operations, has found little attention. The overarching aim of our ongoing work is to find flexible strategies that can enhance the resilience of urban rail systems by explicitly accounting for the uncertainties in the system. Here, we present our initial stages of developing a simulation test bed and testing control strategies for disruption recovery. Moreover, we present the effects of demand uncertainty on the system performance.

\section{Simulation-based disruption analysis}

The starting point is an urban rail simulation testbed consisting of a network with station nodes and line links. In what follows, we describe the basic structure of the simulation, the measure of performance, and a more detailed description of the simulation inputs and optimization approach.

\subsection{Urban Transit System Model}

Every line of the rail network has varying routes that define the exact stop sequence that a train follows when dispatched into the network. Trains operate along their routes according to their assigned trip schedule information, defining the time of first dispatch at their starting station, travel times between stations, as well as dwell times at stations. 
Passenger inflow is controlled according to a specified access rate per origin station. The passenger origin-destination (OD)-matrix consists of the choice probabilities for a particular destination given an origin. When accessing a station, every passenger chooses a destination according to a categorical choice model that incorporates the OD-matrix probabilities. Passenger route choice assumes the shortest-travel-time path between the origin and destination.

All system elements are implemented as agents One agent for every train and passenger, and a single agent for the system controller. The passenger and train agents are able to interact with each other, such that passenger in-vehicle travel times are governed by the train operations (i.e., a train delay will also result in an arrival delay of on-board and waiting passengers). Moreover, the simulation incorporates a disruption generator, that triggers station closures or track disruptions. Station closures do not allow any passenger ingress, egress, or transfer, and trains pass through the station without alighting or boarding passengers. Directional track disruptions cause impassable tracks either along links or at stations, such that any train upstream of the disruption location cannot traverse and pass through, whereas trains downstream of the location can continue to travel.

Incident reports about the location of these disruptions are relayed to the system controller, who makes decisions on ensuing system alerts (i.e., passenger announcements) as well as control actions. The passenger announcements include information on which stations or which lines have been closed. Line closure information includes all routes affected by the track disruptions. The controller can react with control actions. In this analysis, the possible control actions are restricted to adjusting the train headway (i.e., the time interval between consecutive trains) the control parameters are the train dispatch headway and the duration of the schedule adjustment window during which to adjust the headway.

The passengers process the information they receive from the system controller and decide if they either stick with their current itineraries or re-route. These decisions depend on whether a passenger is affected by a disruption and whether re- routing the itinerary is possible. If the passenger is unaffected by a disruption, they will proceed with their current itinerary as planned. However, if the disruption does affect the passenger, one of three options exist: (1) replan their itinerary; (2) proceed to the furthest possible station on the current itinerary; (3) or exit the system at the next possible station. If the original destination or a destination within its vicinity is reachable, the affected passenger will always choose to re-route their itinerary. Only if the destination station is unreachable, the passenger will decide to continue until the furthest reachable station on the current itinerary. If the passenger cannot reach any further on the current itinerary, they will exit the system at the next possible station. Passengers are also able to observe disruptions if they are immediately affected, even if the system controller has not announced any alerts for the particular station or line. For instance, a passenger transferring to a station that just closed, will recognize that the station is closed and will find a different journey itinerary 
according to the same decision criteria mentioned above under items (1) to (3). All other passengers will be unaware of the station closure until the controller announces an alert.

Passengers make egotistic re-routing decisions, that is, re-routing decisions of individual passengers are not influenced by the decisions of other nearby passengers and vice versa. Future and extended work could consider interactions between passengers and how the decision of one passenger affects "neighboring" passengers' decisions.

\subsection{The objective function - Minimization of aggregated delays}

The system level of service is measured as an aggregate travel delay penalty summed over all passengers. The total travel delay penalty $\Psi_{t}$, normalized by the total number of simulated passengers $P$, is computed at the end of the simulation run according to

$$
\Psi_{t}=\frac{1}{P}\left(\sum_{\mathcal{P}_{c}} \psi_{c}+\sum_{\mathcal{P}_{i}} \psi_{i}\right),
$$

where $\mathcal{P}_{c}$ is the set of passengers, who have completed their trip by the end of the, simulation run time, and $\mathcal{P}_{i}$ is the set of passengers, who have not reached their destination by the end of the simulation run time. Respectively, $\psi_{c}$ is the travel delay penalty due to completed passenger trips, and $\psi_{i}$ is the travel delay penalty due to incomplete passenger trips. Since passengers re-route their itineraries according to the disruption information they receive, they may choose to find a new destination $d^{*}$ that is located within a specified radius from the original destination $d$ We thus distinguish between passengers who have reached or are on their way to their original destination and those who have re-routed to a new destination. The contribution to the total travel delay penalty from a completed passenger trip is defined as

$$
\psi_{c}= \begin{cases}\left(t_{o d^{*}}-t_{o d}^{\prime}\right), & \text { if } \quad d^{*}=d, \\ \left|t_{o d^{*}}-t_{o d}^{\prime}\right|, & \text { if } \quad d^{*} \neq d,\end{cases}
$$

where $t_{o d^{*}}$ denotes the actual travel time between origin $o$ and the completed journey destination $d^{*}$, and $t_{o d}^{\prime}$ denotes the expected travel time between the origin and the originally planned destination $d$. If a passenger exits a station that is not the originally planned destination, it can happen that the travel time to the re-routed destination is shorter than the expected. In this case, we assume that the remaining time (i.e., penalty) to get to the originally planned destination is at least the difference between the expected travel time and actual travel time. Hence, we use the absolute value in Eq. (2).

The travel delay penalty due to a passenger trip that has not completed by the end of the simulation run time is defined as

$$
\psi_{i}= \begin{cases}\left(t_{o \bullet}+t_{\bullet} d^{*}-t_{o d}^{\prime}\right), & \text { if } \quad d^{*}=d, \\ \left|t_{o_{\bullet}}+t_{\bullet} d^{*}-t_{o d}^{\prime}\right|, & \text { if } \quad d^{*} \neq d,\end{cases}
$$


where $t_{o_{0}}$ is the current travel time of the passenger when the simulation ends, and $t_{\bullet} d^{*}$ is the expected remaining travel time from the current location to either the re- routed or original destination $d^{*}$

The objective is to minimize the total travel delay penalty by informing passengers about station or line closures so they can re-route their itineraries and implementing control strategies such as adjusting the train headway on selected lines to increase passenger throughput.

\subsection{The simulation inputs and optimization framework}

The simulation builds on the discrete-event simulation library SimPy [13] and is programmed in Python 3.6. The simulation reads in train schedule information from GTFS (General Transit Feed Specification) schedule data [14]. Additionally, station-level passenger inflow data needs to be defined according to the desired time granularity and inflow rate. Similarly, the simulation requires a pre-defined OD- matrix to generate a destination choice for every passenger entering at a specified origin. The route choice model implements the RAPTOR (Roundbased Public Transit Optimized Router) algorithm [15] to generate the shortest travel path between every OD-pair at a given departure time. We modify the RAPTOR algorithm to include station or route-closure information.

The aim of this analysis is to find the optimal parameter values for the train headways to minimize the total time penalty of completed and incomplete trips in the event of a disruption. The optimization process is carried out via a metamodel- based bayesian optimization approach [16]. The metamodel is a multi-dimensional Gaussian Process (GP) regression model [17], with multiple input features and a single output variable. The input features are parameter values for the train headway and control window duration for each of the considered routes. The output is the total time penalty at the end of the simulation run. The Gaussian Process prior covariance is a multi-dimensional Matern kernel [17].

The metamodel-based optimization starts with a defined number of presample points of the parameter values, generated from a Latin-Hypercube design and maxi- min Monte Carlo optimization of the distance between sample points. The simulation is run on these pre-sample points and the GP model is fit to the output. To find the next sample point of parameter values, the method uses an acquisition function that measures the utility of a new sample point by trading off exploring the parameter space with finding the optimal values. In this analysis we define the acquisition function in terms of the expected improvement. The new-found sample point is put through the simulation to generate the next output to be appended to the ensemble of previously determined samples. The optimization process then repeats.

\section{The test network and test cases}

The test network is a reduced set of lines belonging to the New York City (NYC) subway system [18]. The reduced network layout is shown in Fig. 1a. Trains 


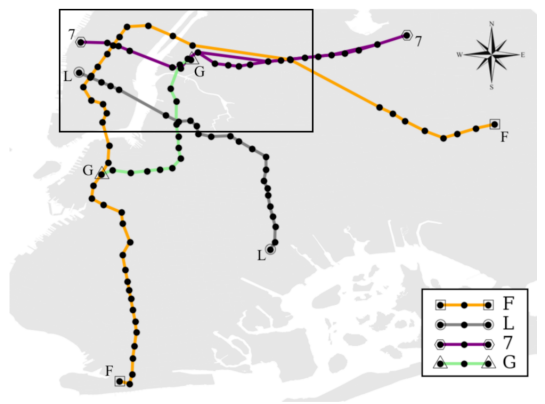

(a)

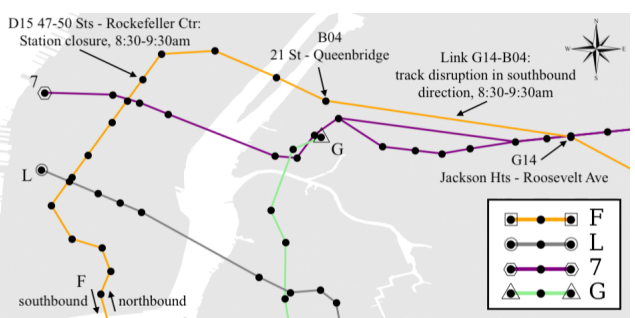

(b)

Fig. 1: Test network consisting of 4 lines and 104 stations. The map in (a) shows the full network layout; (b) shows the boxed frame in (a), indicating the disruption locations and timings.

operate according to the openly accessible trip schedule information provided by the Metropolitan Transport Authority (MTA) of NYC [18]. Some of the trip schedules are modified to be less frequent and account for the reduced set of lines as well as to demonstrate more succinctly the effects of disruption mitigation measures. Passenger inflow data is gathered and processed from the published turnstile counts at every station of the NYC subway system [19]. The actual entry count data are scaled by a factor of $10^{-2}$ to reduce the number of passenger agents and keep computational cost in check. Consequently, train capacities are also reduced by a factor of $10^{-2}$, i.e., from 1500 to 15 passengers. The passenger origin- destination (OD) matrix is based on an estimation model that maps between the historical entry and exit counts to find an estimate for every OD probability coefficient.

The simulation runs a two-hour window, starting at 8:00 am on a typical weekday. During the simulation run time, we trigger an arbitrary unplanned disruption scenario predominantly affecting the southbound direction of line $\mathrm{F}$ in Fig. 1. Namely, a station disruption (i.e., the station is inaccessible by passengers) is reported at 8:30am at station D15 (47-50 Streets - Rockefeller Centre), along with a southbound track disruption (i.e., a disrupted track does not allow any trains to pass through the link) between stations G14 (Jackson Heights Roosevelt A venue) and B04 (21st Street - Queensbridge). The link disruption is unidirectional; that is, the northbound tracks from station B04 to G14 remain in service. Both the station closure and track disruption last for one hour. Fig. 1b shows the locations of the disruptions and their timings.

The controller alerts passengers about the station and line closures 400 seconds after the first incident report is collected, whereas control actions are executed instantaneously when disruptions occur. The particular control actions enforced in this test network involve the train dispatch headway on lines $\mathrm{G}$ and 7 , and the duration of the schedule adjustment. 


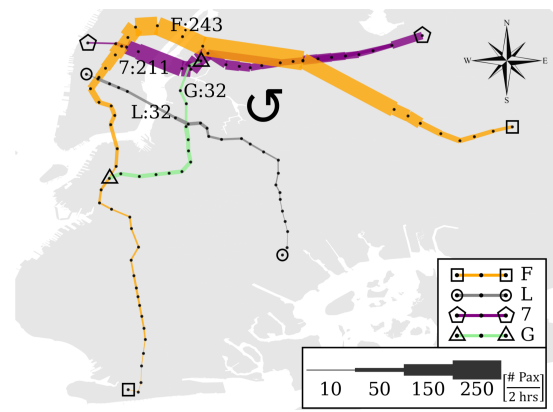

Fig. 2: Southbound link flow levels in the undisrupted network. The circular arrow indicates the southbound flow directions. The indicated numbers are the flow levels (passengers/2-hours) on selected link segments of the F, 7, G, and L line, respectively.

The reduced sub-network and disruption scenario are hypothetical. Simulation results were not validated against a real-world scenario, as reliable real-world data is only available for the full-scale network and slicing count data as well as timetable information corresponding to the sub-component disregards process interactions across the entire full-scale network. Nonetheless, this test case serves to demonstrate the approach and informs scaleable strategies to implement into to the full-scale network.

\section{Results and discussion}

The proceeding results analysis considers four cases. In all cases, the station-level passenger inflow rates are time dependent. The rates vary in 5-minute intervals according to the measured and processed count data at each station.

Case 1 defines the baseline result. It models the undisrupted network and resulting passenger flow, to gain an understanding of the "typical" operations of the network.

Case 2 assesses the impact of the disruption on the passenger link flow levels under two different passenger behavior assumptions: (i) in the first instance, passengers are unaware of any disruptions in the network, and proceed along their itineraries as planned, waiting and travelling on trains as they arrive; (ii) in the second instance, passengers re-plan their itineraries once they either observe a disruption or they receive an alert announcement.

Case 3 uses the passenger re-planning assumption in Case 2 (ii). Additionally, Case 3 assesses how train headway adjustment on selected routes can improve the passenger service level during the disruption, given that passengers will re-route through those routes that are unaffected by the disruption.

Case 4 discusses the effect of passenger demand uncertainty on the system performance. The system is analyzed under the same disruption scenario as in Case 1 through to 3 . However, Case 4 places a negative binomial distribution over the time-dependent station-level inflow rates to introduce an additional noise component. The inflow rates are taken as the mean parameter of the negative binomial distribution. The negative binomial dispersion parameter is fixed to $\phi=0.01$. Another source of demand uncertainty arises from the destination choice model. Every passenger chooses a destination according to a categorical 
distribution, given their origin station and corresponding probabilities in the ODmatrix - In Case 1 through to 3 the random seed for the destination choice model is fixed to produce repeatable samples. Other sources of demand uncertainty, such as the possibility to choose from different route options, or operational uncertainties such as train travel times between stations, are not considered in this analysis.

\subsection{Case 1: The undisrupted network}

The simulated two-hour window results in 1632 passengers trips. Fig. 2 shows the total southbound link flow levels summed over the two-hour simulation window under undisrupted conditions. Under the normal undisrupted operations, the travel delay penalty $\Psi_{t}$ is 54 s per passenger.

\subsection{Case 2: Link flow and travel delay under disruptions}

When modelling the effect of a disruption on the transit system performance, the assumed travel behavior of passengers has significant influence on the resulting aggregate passenger flows and performance metrics. The diagrams in Fig. 3a and $3 \mathrm{~b}$ illustrate the consequences of the two different assumption scenarios during disrupted conditions. Northbound flows are not shown as they are only marginally affected by the disruptions.

By assuming that passengers will not re-plan their itineraries, the southbound link flow on line $\mathrm{F}$ in Fig. 3a noticeably reduces under disrupted conditions. This is owed to the fact that the southbound tracks on line $\mathrm{F}$ are disrupted for an hour, hence not allowing any trains to move southbound upstream of the disrupted link. Since passengers do not re-plan their itineraries, fewer passengers travel through the affected location during the simulated time window. The total travel delay penalty is $\Psi_{t}=775 \mathrm{~s}$ per passenger under no-re-planning.

Conversely, under the assumption that passengers can re-plan their itineraries, the passenger flow re-distributes considerably. Particularly, the total link flow on the green $\mathrm{G}$ line in southbound direction in Fig. 3b noticeably increases. Moreover, the southbound flow on line 7 on link sections parallel to the disrupted section of line $\mathrm{F}$ reduces. Overall, the total travel delay penalty increases to $\Psi_{t}=810 \mathrm{~s}$ per passenger, if passenger re-plan their itineraries.

We note that the travel delay penalty is larger if passengers change their itineraries $\left(\Psi_{t}=810 \mathrm{~s}\right)$, versus holding on to their original itineraries and waiting for the disruption to cease $\left(\Psi_{t}=775 \mathrm{~s}\right)$. On the one hand, this reflects the effects of the model assumptions by virtue of the longer the travel durations along re-planned paths versus the longer wait times at stations. On the other hand, it informs the design of control strategies regarding how to react during the disruption scenario. In fact, these results suggest that recommending to passengers to re-plan their itineraries will result in an overall larger travel delay penalty with the current system schedule and disruption scenario in effect. This is in line with other work which showed that real-time information provision in disrupted networks can result in negative effects on passenger welfare [3]. 


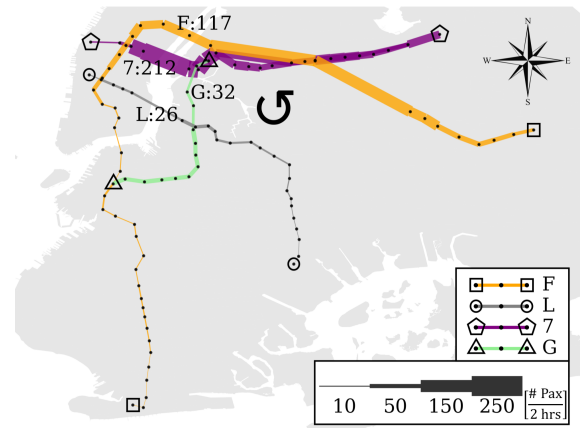

(a) Disrupted network, No re-planning

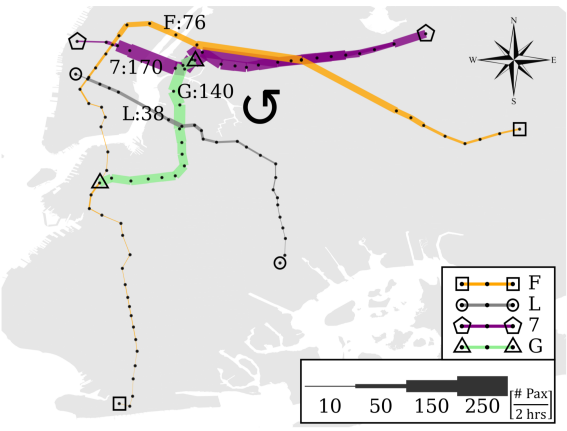

(b) Disrupted network, Re-planning

Fig. 3: Southbound link flow levels under disrupted conditions. Pane (a) shows the link flow under the assumption that passengers stick to their itineraries; Pane (b) assumes that passengers re-plan their itineraries when receiving a disruption alert.

\subsection{Case 3: Optimizing the train headway}

In Case 3, passengers re-plan their itineraries as a result of the controller decision to close line $\mathrm{F}$ in southbound direction and the station closure at 47-50 Streets Rockefeller Centre. We observe that these disruptions along line F cause significant changes in link flow levels along southbound G and 7 trains, due to passengers re-routing their journeys. Moreover, re-routing passenger itineraries result in an increase in the overall travel delay penalty as seen in Section 4.2.

Consequently, our objective is to test whether adjusting the train headway on lines $\mathrm{G}$ and 7 can reduce the travel delay penalty, given that passengers replan their itineraries along these routes. We devise an optimization procedure that aims to minimize the total travel delay penalty per passenger, by finding the optimal adjustment of the train headways on lines $\mathrm{G}$ and 7. The headway is adjusted in both north- and southbound direction, minding that a limited number of trains are available per line and dispatching trains in one direction from a terminal that is not connected to a depot requires that trains arrive in time from the other direction.

We follow the Bayesian simulation-based optimization approach described in Section 2.3. First, we create 5 Latin-Hypercube pre-samples and run the Bayesian optimization routine runs for 100 iterations. The headways can vary between 2 to $20 \mathrm{~min}$. The duration of the schedule adjustment per line and direction is constrained between 0 min to $1 \mathrm{~h}$.

The optimized schedules are illustrated in Fig. 4. It shows the original and new dispatch times of trains in north- and southbound directions along lines $G$ and 7 . For line $\mathrm{G}$, the optimized headways are 2 min over a window of approximately $6 \mathrm{~min}$ in both directions. The adjusted headway on line 7 is approximately 5 min over a 30-min window in northbound direction, and 10 minutes 


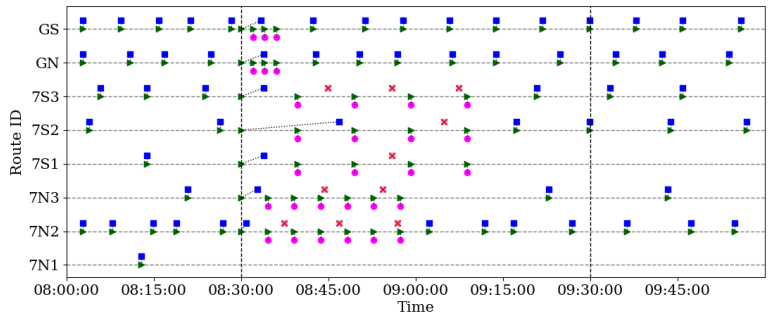

Fig. 4: The optimized train dispatch schedule for lines G and 7 in both northand southbound direction. Route identifiers indicate the line, direction, and additional route stop sequence specification (e.g., "7N2" stands for line 7, northbound, stop sequence 2). For line 7, the headway is adjusted to the same interval on all routes that go in the same direction. Blue squares mark the pre-disruption scheduled dispatch times; magenta circles mark additionally injected trains recommended by the optimization for obtaining the desired headway; red crosses represent trains in the original schedule that are cancelled by the optimization; finally, green triangles mark the actual dispatch times the actual dispatch times can be different from the scheduled or injected dispatch times, given the headway adjustment and possible unavailability of trains at depots; here, all scheduled and injected trains are dispatched as planned, except the first scheduled trains during the disruption window. Vertical dashed lines indicate the start and end of the disruptions on line F.

over a 40-min window in southbound direction. Therefore, the original schedule is restored 40 mins after the disruption. The optimized schedule reduces the total travel delay penalty to $\Psi_{t}=695 \mathrm{~s}$. The optimized schedule and passenger re-planning out-performs the strategy of not recommending to passengers to reroute ( $\Psi_{t}=770 \mathrm{~s}$ with the adjusted schedule), as well as reduces the travel delay penalty with respect to the original schedule $\left(\Psi_{t}=810 \mathrm{~s}\right)$.

\subsection{Case 4: Effects of passenger demand uncertainty}

Fig. 5 plots the variation of the total travel delay penalty $\Psi_{t}$, due to the uncertainty in passenger demand. The boxplots are based on 100 Monte Carlo runs for each of the assumptions in Case 2, assuming either passenger re-planning (Case 2(ii)) or not (Case 2(i)), and Case 3, which assumes passenger re-planning and the optimized schedule. The uncertainty stems from variation in passenger inflow at stations, and the categorical destination choice model. Fig. 5 shows that in all cases the total travel delay considerably varies, independent of whether we assume passenger re- planning or not, or put in effect the optimized schedule. However, the optimized case that flexibly adjusts the train headway overall reduces the total travel delay.

The variation in system performance highlights the flaw of averages [20], and underscores that optimizing the system and schedules should consider the 


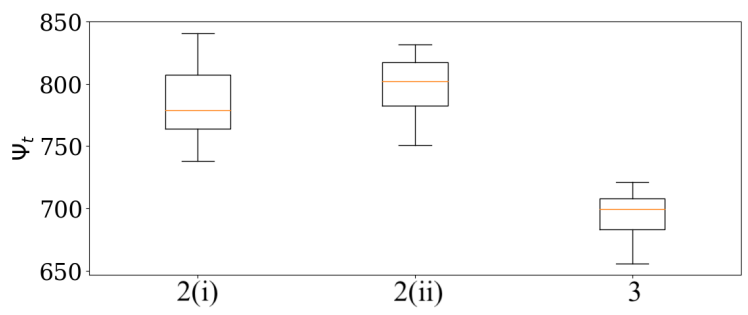

Fig. 5: Boxplots of the system performance uncertainty, measured in terms of the variation of the total travel delay penalty $\Psi_{t}$. The plots differentiate between three scenarios: Case 2(i): The original default schedule and no passenger re-planning; Case 2(ii): The original default schedule including passenger replanning; and Case 3: The optimized schedule including passenger re-planning.

inherent passenger demand uncertainty. Flexible strategies that adapt to the system condition as it evolves could potentially perform better in dealing with this uncertainty. However, this flexibility requires enablers, such as the necessary control structures and system architectures to swiftly implement real-time control actions such as the analyzed headway adjustment or other strategies such as short-turning, expressing, or dead-heading.

\section{Conclusion}

In this work, we present a simulation-based approach to model the dynamic rerouting of passengers and train schedule adjustment during disruptions in an urban rail transport system. Based on a small-scale test network, we demonstrate an optimization procedure to adjust train dispatch schedules and reduce the impact of disruptions. We show that it is possible to reduce the total travel delay penalty by adjusting the train headway on selected train routes. We assume that passengers re- route due to real-time disruption alerts and information, as well as consider rolling stock constraints regarding the available trains for dispatch and train on-board capacities. At last, we show that demand uncertainty considerably changes the resulting travel delay penalty. Nonetheless, flexible adjustment of train headways overall reduces the travel delay even when considering uncertainty. Ongoing work involves finding flexible control strategies that explicitly include the demand uncertainty into the flexible optimization of train operations to further reduce the performance draw-down and augment the recovery process during disruptions.

\section{References}

1. Cats, O.: Dynamic Modelling of Transit Operations and Passenger decisions. PhD Thesis. KTH - Royal Institute of Technology, Sweden (2011) 
2. Cats, O., Koutsopoulos, H.N., Burghout, W., Toledo, T.: Effect of real-time transit information on dynamic path choice of passengers. Transportation Research Record 2217(1), 46-54 (2011)

3. Cats, O., Jenelius, E.: Dynamic vulnerability analysis of public transport networks: Mitigation effects of real-time information. Networks and Spatial Economics 14(34), 435-463 (2014)

4. Cats, O., Jenelius, E.: Planning for the unexpected: The value of reserve capacity for public transport network robustness. Transportation Research Part A 81, 47-61 (2015)

5. Cats, O., West, J., Eliasson, J.: A dynamic stochastic model for evaluating congestion and crowding effects in transit systems. Transportation Research Part B 89, $43-57$ (2016)

6. Othman, N.B., Legara, E.F., Selvam, V., Monterola, C.: A data-driven agent-based model of congestion and crowding effects in transit systems. Journal of Computational Science 10, 338-350 (2015)

7. Yin, H., Han, B., Li, D., Wu, J., Sun, H.: Modeling and simulating passenger behavior for a station closure in a rail transit network. PLoS One 11(12), e0167126 (2016)

8. Kroon, L., Maróti, G., Nielsen, L.: Rescheduling of railway rolling stock with dynamic passenger flows. Transportation Science 49(2), 165-184 (2014)

9. Hurk, E.v.d., Kroon, L., Maróti, G.: Passenger advice and rolling stock rescheduling under uncertainty for disruption management. Transportation Science 52(6), 13911411 (2018)

10. Ghaemi, N., Zilko, A.A., Yan, F., Cats, O., Kurowicka, D., Goverde, R.M.P.: Impact of railway disruption predictions and rescheduling on passenger delays. Journal of Rail Transport Planning \& Management 8, 103-122 (2018)

11. Zhu, Y., Goverde, R.M.P.: Railway timetable rescheduling with flexible stopping and flexible short-turning during disruptions. Transportation Research Part B 123, 149-181 (2018)

12. Cacchiani, V., Huisman, D., Kidd, M., Kroon, L., Toth, P., Veelenturf, L., Wagenaar, J.: An overview of recovery models and algorithms for real-time railway rescheduling. Transportation Research Part B 63, 15-37 (2014)

13. Scherfke, S., Lnsdorf, O., SimPy, https://bitbucket.org/simpy/simpy/src/default/. Last accessed 23 May 2019

14. The General Transit Feed Specification, https://www.gtfs.org/. Last accessed 23 May 2019

15. Delling, D., Pajor, T., Werneck, R.F.: Round-based Public Transit Routing. Transportation Science 49(3), 591-604 (2014)

16. Frazier, P.I.: A tutorial on Bayesian Optimization. arXiv 1807.02811v1, 1-22 (2018)

17. Rasmussen, C.E., Williams, C.K.I.: Gaussian processes for machine learning. MIT Press, Cambridge MA, USA (2006)

18. Metropolitan Transport Authority, MTA Static Data Feeds, http://web.mta.info/developers/developer-data-terms.htmldata. Last accessed 23 May 2019

19. Metropolitan Transport Authority, Turnstile Data, http://web.mta.info/developers/turnstile.html. Last accessed 30 August 2018

20. Savage, S.: The Flaw of Averages, San Jose Mercury News (2000) 